\title{
Community Neurorehabilitation: A Synthesis of Current Evidence and Future Research Directions
}

\author{
Sarah E. Chard \\ Department of Sociology and Anthropology, University of Maryland, Baltimore County, Baltimore, Maryland 21250
}

\begin{abstract}
Summary: Over the past decade, community neurorehabilitation has emerged as a promising extension of neurological rehabilitation. The goal of community neurorehabilitation is to maximize functional ability and quality of life through multidimensional rehabilitation that occurs while the individual is living in a home versus acute or transitory care setting. Because of its multidisciplinary focus, many variations of community neurorehabilitation teams have been implemented. Critical gaps exist, however, in understanding of the influence of structural and procedural differences among programs, as well as patient level variables such as social support, on recovery. This paper
\end{abstract}

examines the current evidence of the effectiveness of community neurorehabilitation through a review of the findings of systematic reviews and meta-analyses of four neurological conditions: stroke, multiple sclerosis, traumatic brain injury, and Parkinson's disease. It focuses in particular on the data regarding physical therapy and occupational therapy, which are two of the primary components of community neurorehabilitation programs. Key Words: Community neurorehabilitation, review, stroke, multiple sclerosis, traumatic brain injury, Parkinson's disease.

\section{INTRODUCTION}

Community neurorehabilitation is receiving increasing attention from researchers and health policy analysts who seek to determine whether rehabilitation that is provided in home or community settings is effective for chronic neurological conditions. This paper presents a qualitative synthesis of community neurorehabilitation systematic reviews for four leading neurological disorders: stroke, multiple sclerosis, traumatic brain injury, and Parkinson's disease. Systematic reviews, particularly meta-analyses, provide the most statistically powerful evidence regarding community neurorehabilitation; they are critical for assessing the state of the neurorehabilitation field because of the limited number of large, high quality randomized controlled evaluations. After noting key developments, this examination then identifies gaps in current approaches and offers suggestions for future research directions.

The role of rehabilitation in the treatment of neurological disorders is an increasingly important issue given

Address correspondence and reprint requests to: Sarah E. Chard, Ph.D., Department of Sociology and Anthropology, University of Maryland, Baltimore County, 1000 Hilltop Drive, Baltimore, MD 21250; Ph: 410-4553380; Fax: 410-455-1154. E-mail: schard@umbc.edu. the prevalence of neurological disorders and related impairments. In 2003, the U.S. prevalence for stroke was 5,500,000, with 500,000 new strokes and 200,000 recurrent strokes occurring each year. ${ }^{1}$ Although the rate of stroke deaths has fallen $18.5 \%$ over the past decade, over $1,100,000$ individuals in the U.S. report on-going functional impairments as a result of a stroke. ${ }^{1}$ Traumatic brain injury is similar to stroke in that it also can result in chronic disability. The annual incidence of traumatic brain injury is roughly 1.4 million, with 80,000 to 90,000 individuals experiencing long-term disability as a result of their injury. ${ }^{2}$

Although progressive neurological diseases, such as Parkinson's disease and multiple sclerosis are less common than stroke and traumatic brain injury, they still are a major source of the neurological disease burden of this country. Estimates on the prevalence of Parkinson's disease vary widely, ranging from $500,000^{3}$ to 1.5 million, with approximately 60,000 new cases occurring each year. ${ }^{4}$ Finally, the prevalence of multiple sclerosis is likewise difficult to estimate, but between 250,000 and 350,000 individuals have been clinically diagnosed with the disease. ${ }^{5}$ Thus, neurological disorders like stroke and traumatic brain injury and progressive neurological diseases like Parkinson's disease and multiple sclerosis all may result in costly chronic impairments that are cur- 
rently the subject of much community neurorehabilitation research.

Community neurorehabilitation refers to specialized, neurological rehabilitation for individuals living in their home community, as opposed to inpatients of acute care or rehabilitation institutions. As a branch of neurorehabilitation, community neurorehabilitation serves three broad functions. First, it allows for earlier, coordinated inpatient discharge. ${ }^{6,7}$ Second, it provides a mechanism for rehabilitation for individuals who do not require intensive hospitalization or who have been discharged from an inpatient setting but who would benefit from further therapy. ${ }^{8}$ Third, it supplies periodic, maintenance rehabilitation for individuals with chronic, progressive neurological disorders, but who again, do not require hospitalization. $^{9}$

This growing field is grounded in a belief in the importance of on-going rehabilitation for neurological illness; i.e., that neurorehabilitation can be beneficial even years after an injury or illness event ${ }^{10}$ and that long-term and recurrent rehabilitation may help individuals maintain or advance their functional status. ${ }^{11,12}$ It is further argued that such community maintenance rehabilitation does not require the intensive and expensive care of an acute, inpatient hospital, but rather, specialized rehabilitation can be provided on an outpatient basis. ${ }^{8,13}$ The effectiveness of this care, especially compared with inpatient treatment, remains a central question for the field.

Community neurorehabilitation also is premised on the view that disability is a multidimensional construct of the social environment, as proposed by the World Health Organization's International Classification of Functioning, Disability, and Health (ICF). ${ }^{14,15}$ As such, much of community neurorehabilitation emphasizes the importance of promoting wellness by improving function and quality of life, as defined once individuals reside in their home community. This perspective further recognizes that rehabilitation needs change over the course of an illness and as patients adjust to their postacute environment. ${ }^{16,17}$ To identify patients' needs within the home community, neurorehabilitation programs often utilize a patient-centered approach that establishes and modifies therapeutic goals through dialogue among a therapist or therapeutic team, the patient, and the patient's caregivers. ${ }^{18,19}$ Rehabilitation protocols, therefore, are individualized to each patient, with the common goal of developing the patient's ability to function within his or her unique social and physical environment through therapeutic interventions, education, support, and environmental modifications.

While individual physical or occupational therapists may provide home-based services, often as an outreach program of a hospital, ${ }^{8}$ a more widely held position is that community neurorehabilitation requires a multidisciplinary decision-making "team" in order to develop and provide effective holistic treatment. ${ }^{20,21}$ Multidisciplinary programs, which may be either affiliated with or independent from local hospitals, frequently incorporate general practitioners, neurologists, psychologists, nurses, physical, occupational or vocational therapists, and social service professionals, among others. The precise composition and structure of multidisciplinary community neurorehabilitation programs, however, is not consistent and programs vary considerably in their purpose, design, and operationalization.

In the United Kingdom, for example, where community-based care models have been widely implemented, recent surveys suggest programs differ according to their focus within the rehabilitation process; e.g., rehabilitation teams may concentrate on providing early discharge support, after discharge therapy, "late community rehabilitation," or general-practitioner guided therapy. ${ }^{22}$ Others note that programs may specialize in specific neurological conditions or care for neurological patients as a de facto result of the age of their target clients (e.g., older adults). ${ }^{23,24}$ Survey results suggest community programs can range in size from 2.2 to 15.3 full time equivalent staff, with physical therapists and occupational therapists among the most common members, and psychological and social work staff the least. As a result of these inconsistencies, there is concern that some programs may lack certain expertise, especially in dealing with psychological disorders. ${ }^{25}$

The variability across programs and interventions complicates the establishment of a clear evidence base for the effectiveness of community neurorehabilitation. ${ }^{11,26}$ Although the ICF, in part, is an attempt to create a standard terminology for rehabilitation services, no uniform classification system for rehabilitation interventions and outcomes, including community rehabilitation and community neurorehabilitation, is in use. ${ }^{27}$ As Turner-Stokes et al. ${ }^{28}$ found in their review of acquired brain injury rehabilitation research, the lack of standardization means that "it is probable that the actual content of any two programmes within the same category varied greatly, and also that similar programmes may have been given different labels." Thus, community neurorehabilitation programs and clinical trials often differ in their structure and operation as well as in the language used to characterize interventions. As a result, identifying and evaluating similar therapies across clinical trials can be challenging.

Further limiting the establishment of the evidence base for community neurorehabilitation is that many clinical trials, to date, have tended to focus on single-discipline interventions and outcomes, despite widespread acknowledgement of the importance of multidisciplinary approaches. $^{20,29}$ This paper, therefore, examines the evidence regarding multidisciplinary programs and community-based single disciplines, which serve as the 
building blocks of multidisciplinary programs. Ultimately, however, for community neurorehabilitation to move forward there is a need for studies on the effects of collaborative, multidisciplinary care and the impact of variations in the structure and process of such programs.

Community neurorehabilitation has developed over the past decade as an extension of neurorehabilitation that addresses an individual's needs that emerge in the postdischarge, home environment. The effectiveness of this approach across neurological disorders is not well established. However, as this review demonstrates, evidence is slowly accumulating for several neurological disorders that suggests community neurorehabilitation may play an important role both immediately upon release from an acute care setting and later as individuals seek to maintain functioning and social participation.

\section{METHODOLOGY}

The author identified systematic and meta-analytic studies through searches of three electronic databases, MEDLINE (1966-2006), the Cochrane Database of Systematic Reviews, and Academic Search Premier (a social science database); existing bibliographies; and consultation with experts. Three primary search strings were employed with the electronic databases: 1) "neurorehabilitation and (community or outpatient)"; 2) "neurological and rehabilitation and (community or outpatient)"; and 3) "(neurorehabilitation or rehabilitation) and (review or meta*) and (stroke or multiple sclerosis or Parkinson's or brain injury)." The search results were examined for meta-analyses and qualitative and quantitative systematic reviews of community (or outpatient) neurorehabilitation or neurological rehabilitation. This broad search strategy and inclusion criteria allowed the author to capture the range of definitions, methodologies, and interventions involved with community neurorehabilitation.

\section{RESULTS}

Much of the research on community neurorehabilitation has focused on the effectiveness of interventions for stroke. Fewer studies and reviews have examined the role of community neurorehabilitation for multiple sclerosis, brain injury, and Parkinson's disease. For example, Medline contains 118 entries for stroke and neurorehabilitation or neurological rehabilitation; it contains only 48 entries for multiple sclerosis using the same search strategy, 83 for brain injury exclusive of stroke, and 18 for Parkinson's disease. Likewise, when looking specifically at review articles and meta-analyses for community (or outpatient) rehabilitation or neurorehabilitation, MEDLINE contains 72 entries for stroke, 6 entries for multiple sclerosis, 46 for brain injury excluding stroke, and four for Parkinson's disease. This bias likely reflects in part the differences in the prevalence of stroke versus other neurological conditions; however, it severely limits the evidence base regarding the effectiveness of community rehabilitation across neurological conditions.

\section{Stroke}

There are several high quality meta-analyses and systematic reviews that address the safety and effectiveness of community neurorehabilitation for stroke. One of the largest is the Outpatient Service Trialists review, which examines the impact of home-based outpatient rehabilitation services within one year of hospital discharge or stroke onset for stroke patients living at home. ${ }^{11,30,31}$ The authors analyze 14 randomized controlled trials of outpatient occupational therapy, physical therapy, or multidisciplinary interventions providing task-oriented skills. They find that rehabilitation participation reduces the likelihood of deterioration in patients' ability to complete activities of daily living; i.e., "the absolute reduction in risk of deterioration in ability to undertake activities of daily living was seven per 100 patients allocated therapybased rehabilitation". ${ }^{30}$ Due to insufficient data, no conclusions could be drawn on the impact of therapies on patient quality of life, subsequent hospital admissions, or long-term institutional care.

Other systematic reviews focus on multidisciplinary community neurorehabilitation and "early discharge programs," arguing that multidisciplinary early discharge programs safely and effectively lower the number of inpatient hospital treatment days. ${ }^{17}$ Indeed, the evaluation by Langhorne et al. ${ }^{6,7,32}$ of 11 randomized controlled trials concludes that multidisciplinary early stroke discharge teams reduce inpatient hospital stays by 8 days. Participants in such programs have a decreased risk of death and long-term institutionalization. Langhorne et $\mathrm{al}^{7}$ suggest the greatest effects occur in cases of mild to moderate disability.

Studies examining the impact of single disciplines, such as occupational therapy, have more mixed results. Steultjens et al., ${ }^{26}$ for example, distinguish 6 types of occupational therapy: "1) training of sensory-motor functions; 2) training of cognitive functions; 3) training of skills such as dressing, cooking a meal, or performing domestic activities; 4) advice and instruction on the use of assistive devices; 5) provision of splints and slings; and 6) education of family and primary caregivers." Based on seven studies, they report that comprehensive occupational therapy, which includes all 6 types of occupational therapy, is associated with moderate, but statistically significant, improvements in primary outcome measures of "activities of daily living, extended activities of daily living, and social participation and the secondary process measures of arm and hand function, muscle tone, and cognitive functions". 
Steultjens et al. ${ }^{26}$ also evaluate the evidence regarding each occupational therapy category. Based on 8 skill training studies they suggest minimal support for the impact of skill training occupational therapy on activities of daily living; their review of four cognitive training studies demonstrates slight improvement in visual-spatial ability. The data regarding training of sensory-motor function, assistive device instruction, splint use, and caregiver education are inconclusive due to the lack of high quality studies.

In their meta-analysis of the impact of occupational therapy for patients with stroke, Walker et al. ${ }^{33}$ likewise identify significant effects from community-based occupational therapy. The authors conduct an individual level meta-analysis of the impact of 8 randomized community occupational therapy trials and report that after adjusting for age and baseline dependency, community occupational therapy participants increased their extended activities of daily living score. There were no effects, however, on death or psychiatric status as measured by the general health questionnaire.

The recent systematic analysis by Van Peppen et al. ${ }^{34}$ of 151 stroke physical therapy studies similarly provides qualified support for specific neurological rehabilitative therapies. The authors conclude that task-oriented physical therapy tends to improve activities of daily living scores. In addition, while impairment-focused therapy (e.g., exercise to encourage muscle growth) does strengthen muscles, this improvement does not result in a change in activities of daily living. Other meta-analyses suggest that aerobic exercise interventions increase aerobic capacity, walking speed, and walking endurance. ${ }^{35}$ The ability of aerobic physical therapy to reduce the risk of secondary diseases, such as cardiovascular disease, needs further exploration. ${ }^{35}$

There currently is no clear evidence, however, that community-based programs should replace hospital admission for stroke. ${ }^{36} \mathrm{~A}$ recent analysis of four randomized controlled trials and quasi-randomized controlled trials of a "hospital at home" intervention found that there were no differences in the extended activities of daily living, subjective health status, caregiver stress, death, or "death or institutionalization" rates in the studies, but there were a slightly higher number of deaths and lower outcomes among the home-care groups.

\section{Multiple sclerosis}

Neurorehabilitation for multiple sclerosis often requires attention to highly individualized, disparate symptoms that range from fatigue and spasticity to cognitive impairments to reduced bladder and bowel control. ${ }^{37}$ The irregular variations in the course of the disease can make sample selection criteria, outcome measures, and study comparisons problematic. ${ }^{38}$ Despite the difficulties investigating multiple sclerosis community neurorehabili- tation, research suggests that community-based multidisciplinary neurorehabilitation and community physical therapy rehabilitation can reduce the physical and psychological effects of the disease.

Although Kesselring and Beer ${ }^{39}$ advocate for inpatient neurorehabilitation to reduce the logistical burden on patients, caregivers, and therapists, their qualitative review indicates that multidisciplinary rehabilitation in either setting is effective in helping patients adapt to changes in health status and function. They suggest two major randomized controlled trials of outpatient, multidisciplinary teams that provided counseling support, education, and/or physical and occupational therapy resulted in improved fatigue level and functional abilities. $^{40,41}$

In contrast to the studies on multidisciplinary rehabilitation, the review by the Cochrane Multiple Sclerosis Group of the evidence regarding occupational therapy for multiple sclerosis is unable to determine whether community-based occupational therapy, specifically energy conservation and counseling, is effective for multiple sclerosis. ${ }^{42}$ Their lack of conclusion primarily is due to the limited number of randomized controlled trials. Further study of occupational therapy and multiple sclerosis is warranted, however, because the 2 energy conservation studies that were included in the review found that the intervention reduced fatigue; the counseling intervention had no statistically significant impact.

Other recent reviews do find support for the use of community-based "exercise" therapy. In this case, the examination by the Cochrane Multiple Sclerosis Group of nine randomized controlled trials, which defines exercise broadly to include rehabilitation, physical therapy, and training, suggests that exercise therapy is safe, and can improve aerobic capacity, strength, functional independence measure scores, and to a lesser degree, psychological status. ${ }^{12}$ There is no evidence that 1 exercise modality is more effective than others. The qualitative reviews by Brown and $\mathrm{Kraft}^{9}$ and Thompson ${ }^{19}$ similarly conclude that outpatient exercise interventions improve disability, quality of life, and strength. Exercise also helps preserve cardiovascular function but does not necessarily advance fitness levels.

Many of the studies reviewed in these analyses, however, involve individuals with some mobility. There is a gap in knowledge of the effects of rehabilitation on individuals who are not mobile and a need for additional research to determine with greater specificity the intensity, duration, and frequency of rehabilitative exercise required to produce an effect. ${ }^{9,12,43}$

As a result of this literature there is consensus that individuals with multiple sclerosis should not avoid activity as previously advised; rather, after an initial assessment, an exercise plan that is appropriate for an 
individual's functional capacity and susceptibility to overheating should be developed. ${ }^{9,44}$

\section{Traumatic brain injury}

Although traumatic brain injury has some parallels to stroke and multiple sclerosis in the heterogeneity of its symptoms, several characteristics distinguish it from other neurological conditions. First, traumatic brain injury can result in particularly high levels of mental and behavioral changes. ${ }^{45}$ Neurorehabilitation teams must address difficulties in self-appraisal and unrealistic activity expectations in addition to physical disorders. Second, traumatic brain injury tends to occur in a more diverse age population than other neurological conditions. Community neurorehabilitation for traumatic brain injury therefore must address the wide-ranging needs, goals, and responsiveness of children, adults, and older adults.

There are a limited number of randomized controlled trials and subsequent systematic reviews for traumatic brain injury. One of the most comprehensive, the analysis by the Cochrane Injury Group of inpatient and outpatient traumatic brain injury rehabilitation concludes, based on two high quality controlled studies of community multidisciplinary rehabilitation programs, that there is "limited evidence" that such outpatient programs can advance function; i.e., the ability to complete a task or action, particularly when interventions are directed toward an explicit objective. ${ }^{28}$ At the same time, they also find "limited evidence" from two methodologically lower quality studies that "specialist inpatient rehabilitation" may have a greater effect on function than "local" or "home-based advisory services." They caution, however, that further research is warranted given the potential selection bias between the treatment and control groups. $^{28}$

The review by the Cochrane Injury Group additionally finds "strong evidence" that higher intensity inpatient rehabilitation for stroke and other types of traumatic brain injury can result in quicker functional improvement. Their analysis of inpatient and out patient studies further suggests there is "strong evidence" that mild traumatic brain injury patients who experience less than one hour of amnesia typically do not require rehabilitation, patients with over 1 hour of amnesia benefit from routine follow-up that includes information and counseling, and individuals with "moderate to severe injury" do profit from a higher level of intervention. The authors warn that such individuals may not present themselves for assistance without routine follow-up. ${ }^{28}$

A qualitative analysis of quasi-experimental studies finds a broader range of effects from outpatient neurorehabilitation, suggesting such programs are associated with advances in psychological status, residence and employment or education status, community residence, em- ployment, and school enrollment. ${ }^{46}$ Others indicate that specific types of community therapy can effectively address specific symptoms; e.g., cognitive rehabilitation involving personal electronic devices (e.g., alarms) can assist with memory failure and compensatory cognitive rehabilitation may promote social connections and selfappraisal. ${ }^{47}$ It must be cautioned, however, that in the absence of randomized controlled trials it can be difficult to establish causality; e.g., the role of therapy versus spontaneous recovery. ${ }^{48}$

\section{Parkinson's disease}

Similar to traumatic brain injury, there is a critical need for quality research regarding the effectiveness of neurorehabilitation, especially community neurorehabilitation, for Parkinson's disease. ${ }^{29,49}$ One comprehensive analysis, for example, examines 6 Cochrane systematic reviews to determine the effect of speech and language therapy, physical therapy, and occupational therapy for Parkinson's disease, but these authors do not distinguish the efficacy of community versus inpatient neurorehabilitation. ${ }^{50}$ They do suggest, however, that each of the 23 randomized controlled trials in the Cochrane reviews contains at least one serious methodological or analytical flaw; furthermore, there are few consistent outcome measures. As a result, their conclusions are very restricted, arguing the only clear evidence is that physical therapy improves participants' walking speed and stride, while more limited support exists for the use of cueing to improve walking characteristics. ${ }^{50,51}$ The extent to which laboratory cueing methods apply to the home environment is unknown. ${ }^{51}$

There also are mixed findings on the effectiveness of occupational therapy. The comprehensive review by Deane et al. ${ }^{50}$ suggests it is not possible to determine the effect of occupational therapy because of the small number of studies and problems with trial design. One of the few meta-analyses of 16 outpatient-clinic and homebased occupational therapy interventions involving both general and task-specific occupational therapy, however, did conclude that occupational therapy interventions show "success rates of $37 \%$ for control group members as opposed to $63 \%$ success rates for intervention group members". 52

Finally, there is evidence that speech-language therapy for dysarthria improves voice volume; caregivers also indicate that Lee Silverman Voice Therapy improves clarity, with some measures of quality lasting two years postintervention. ${ }^{50}$ At the same time, studies on Parkinson's disease show limited effect on quality of life, suggesting that discrepancies exist between clinical outcomes and outcomes that are meaningful to the patient. ${ }^{50}$

Thus, there is only modest evidence of the effectiveness of community neurorehabilitation for Parkinson's disease. The lack of evidence is in large part because of 
the lack of high quality, randomized controlled trials. Further exploration is warranted, however, as a recent broader analysis that, unlike the studies above, incorporated 44 observational, quasi-experimental and randomized controlled studies, found small but consistent evidence for the effectiveness of multidisciplinary approaches as well as single-discipline programs involving physical therapy, occupational therapy, speech and language therapy, counseling, or education. ${ }^{29}$ The randomized, controlled crossover studies by Trend et al. ${ }^{53}$ and Wade et al. ${ }^{54}$ in particular found outpatient multidisciplinary programs resulted in improved mobility, speech, and health-related quality of life for individuals with Parkinson's disease and mental health benefits for their caregivers. With the exception of mobility, however, these gains did not remain at 6 months, which highlights the need for research on the potential role for long-term, periodic maintenance rehabilitation in cases of Parkinson's disease.

\section{DISCUSSION}

Although inadequate trial designs and inconsistent outcomes limit the conclusions of reviews, evidence is accumulating in support of the effectiveness of community neurorehabilitation across neurological conditions. Qualitative and quantitative systematic reviews and meta-analyses indicate community-based physical therapy and occupational therapy have a positive influence on functional skills; i.e., activities of daily living for individuals with stroke, multiple sclerosis, and traumatic brain injury. While less high quality data exist regarding Parkinson's disease, the results of several observational trials hint at further productive research opportunities. One striking finding across these studies is the effectiveness of community physical and occupational task-oriented therapies. ${ }^{34}$ Thus, similar to inpatient neurorehabilitation, community neurorehabilitation that focuses on the completion of specific activities rather than reducing physical impairments, shows notable promise. ${ }^{14}$

These reviews contain several other key findings that challenge previous conclusions regarding specific disorders. First, it is now clear that coordinated early discharge stroke programs are as safe and effective as inpatient programs. ${ }^{7,55,56}$ Second, physical activity for multiple sclerosis is not linked to disease exacerbation; rather, when carefully conducted (e.g., limiting body temperature increases), physical therapy can reduce fatigue and improve mental status. ${ }^{9,19,44}$ Third, the intensity of an intervention may influence recovery in cases of stroke and traumatic brain injury, especially among young adults. ${ }^{28}$

The evidence regarding the intensity or dose of community neurorehabilitation is especially noteworthy given recent inpatient research that calls for a reassessment of theories regarding the role of therapeutic inten- sity. ${ }^{57}$ While the Post-Stroke Rehabilitation Outcomes Project $^{58}$ has several methodological limitations, the researchers report a positive association between the intensity (i.e., length in minutes) and focus of inpatient therapies and functional measures at discharge. ${ }^{59}$ Although the meta-analysis by Kwakkel et al. ${ }^{60}$ of inpatient and outpatient rehabilitation suggests the effects of such increased physical and occupational therapy may be small, even single point changes in Barthel scores are clinically significant. Others argue, based on analyses of community occupational therapy, that a one-point increase in an individual's extended activities of daily living score indicates mastery of an activity such as walking outside or participating in household operations. ${ }^{33}$ Furthermore, a very recent study finds greater quantities of outpatient therapy for stroke can lead to increases in social participation and overall activity, and lower rates of depression. ${ }^{61}$

Community neurorehabilitation reviews that find no high quality evidence in support of an intervention, however, should not be interpreted as suggesting that interventions are ineffective or of no use to patients, as Chesnut et al. ${ }^{47}$ argue in their review of the traumatic brain injury literature. Rather, there is a need for additional research before conclusions can be drawn. ${ }^{50}$ Furthermore, limited evidence that comes from lower quality studies can still be used to advise patients on the potential for a positive impact from neurological rehabilitation. $^{52}$

This synthesis of the evidence regarding community neurorehabilitation also reveals a major limitation in current approaches to studies of effectiveness. Many community clinical trials and subsequent systematic reviews tend to focus on evaluating therapeutic outcome without appraising the impact of structural and process variables, and potential confounding by patient level variables. Outcome measures likewise tend to narrowly focus on function, but not necessarily an individual's participation and quality of life, as recommended by the ICF. ${ }^{15}$ The constricted assessments found in community neurorehabilitation research is troubling given the emphasis of neurorehabilitation on individualized protocols and goals, ${ }^{18,20}$ and the tremendous variation in outpatient trial settings, staffing, management, and therapy implementation. ${ }^{14,42}$ There may be little documentation of these variations within a clinical trial and limited analyses of the impact on patient recovery. ${ }^{61,62}$ As a result of this gap it can be difficult to distinguish the effects of specific elements of multidisciplinary interventions; e.g., the impact of physical therapy versus social-psychological components of a trial, ${ }^{39}$ as well as how therapy combinations act together to produce effects beyond each individual therapy. Indeed, the conclusions of the analysis by the Outpatient Service Trialists ${ }^{30}$ of stroke services applies to many systematic reviews, "the exact 
nature and content of therapy-based rehabilitation services is not answered by our review; neither is the most effective way to structure provision of these services nor their economic benefits. What does seem clear is that the debate should move from whether such services are effective to how to make the most of their benefits".

One model that could contribute to understanding of the steps needed to maximize the effectiveness of community neurorehabilitation is the "structure-process-outcome" model of healthcare delivery. ${ }^{63}$ This model, which has been widely used to evaluate healthcare services, suggests that the structure and process of care impact the provision of "quality" medical, or in this case, rehabilitative, care. Furthermore, it is hypothesized that healthcare structure determines the process of care, which in turn influences outcome. ${ }^{63,64}$ Through application of the "structure-process-outcome" model of health care, researchers could more clearly discern the degree of heterogeneity among outpatient interventions in terms of their design and implementation in homes and community facilities, the level and impact of individualization within a trial, and the appropriateness of cross-trial comparisons.

Within rehabilitation research, the limited studies that have implemented this model have operationalized structure to include the types, training, and number of fulltime equivalent staff, the workload, the characteristics of the physical plant (e.g., presence of an adaptive kitchen or bathroom), and the organizational setting; e.g., number of beds and additional services. ${ }^{65}$ Process variables include the minutes spent per day in specific physical, occupational, or speech-language therapy activities (e.g., bed mobility, bathing, or problem solving), ${ }^{59}$ the coordination among staff, ${ }^{6}$ and the therapist-patient relationship or "working alliance". 66

While efforts are underway to establish a reliable and valid standardized measure of the structure and process of rehabilitation services, ${ }^{67}$ several inpatient and outpatient rehabilitation research projects support further use of this model. In their analysis of inpatient stroke care at Veterans' Administration medical centers, for example, Hoenig et al. ${ }^{68}$ report that structure variables, such as staff organization, influence the care process and that the process of care correlates with 6-month outcome measures of function. The findings of Hoenig et al. ${ }^{68}$ are particularly striking because they suggest "strong systemic organization may be able to offset some of the adverse effects of low staff expertise."

The Post-Stroke Rehabilitation Outcomes Project takes a very detailed look at the process of inpatient rehabilitation care by measuring the specific number of minutes spent in therapeutic activities. ${ }^{59}$ As noted above, they report that the time spent within a therapeutic session on higher level goals correlates with an individual's functional scores at discharge.
In a recent study examining the structure and process of care for outpatient rehabilitation, Hankey and Langhorne $^{6}$ indicate that better recovery rates occur among participants in coordinated multidisciplinary early discharge programs versus uncoordinated rehabilitation programs. A qualitative study by Hart, ${ }^{69}$ on the experience of stroke, likewise links system disorganization and communication processes and declines in recovery. Hart argues it is not possible to understand the experience of living with stroke in isolation from the experience of interacting with health and social care services: the interplay between experience and system impacts on the disease process itself.

Similarly, Klonoff et al. ${ }^{66}$ report process variables, such as staff rapport with patients, the degree of congruence in care expectations, and patient attendance and adherence, are associated with postrehabilitation employment or school enrollment. In marked contrast to the above findings, however, the detailed analysis by Logan et al. ${ }^{70}$ of the Trial of Occupational Therapy and Leisure suggests that specific outpatient occupational therapy interventions do not necessarily correlate with expected outcomes. As these studies demonstrate, examination of the multiple dimensions of the structure and process of rehabilitation reveals additional variables to consider in the development and evaluation of the evidence base of community neurorehabilitation.

Attention to the third dimension of this model, therapeutic outcome, also could lead to a broader understanding of the impact of rehabilitation. Currently, there is a necessary reliance on standardized measures of disability and function; however, many of these instruments have serious limitations. The Expanded Disability Status Scale is a common measure of disability; however, critics charge it is an "insensitive" measure of the effects of multiple sclerosis, failing to capture fatigue levels and changes in social roles that heavily impact quality of life. ${ }^{9}$ Barthel's Index likewise is more effective at measuring basic activities of daily living, rather than instrumental activities of daily living and social interaction, ${ }^{71}$ and the clinical significance of current score groupings is questioned. ${ }^{72}$ Others argue that a focus on functional outcomes obscures the psychosocial benefits of therapy, especially home-based sessions. ${ }^{73}$ In addition, although improvements in standardized functional scores can indicate meaningful life skill increases, as discussed above, an association with better quality of life cannot be assumed. Cicerone et al. ${ }^{74}$ discovered, for example, that increased community involvement among participants in a traumatic brain injury intervention did not result in participants' higher satisfaction with their community function status. Participants' perceptions of their deficits and the meanings attached to functional improvements or losses influence quality of life reports. Thus, the limits of current outcome measures need to be acknowledged 
more frequently within studies and a relationship between functional improvement and quality of life should not be supposed. Rather, there is a need for consistent use of separate quality of life instruments, including qualitative assessments of participants' perceptions of therapeutic effects. ${ }^{73}$

Finally, as the work by Cicerone et al. ${ }^{74}$ suggests, the role of psychosocial variables deserves special consideration in research design. Few studies and systematic reviews of community neurorehabilitation examine possible confounding by psychosocial patient level variables. This is despite the evidence that variables such as social support, ${ }^{75}$ self efficacy beliefs, ${ }^{76,77}$ and patient and caregiver engagement or motivation ${ }^{78,79}$ play a role in rehabilitation adherence or stroke recovery. Studies that do include patient psychosocial resources tend to measure it one-dimensionally; e.g., social support is operationalized in terms of the existence of a caregiver rather than as a multifaceted construct. ${ }^{6,32}$ Mental status assessments tend to involve measures of mood rather than beliefs and motivations. ${ }^{59}$ When incorporated as a dependent variable, psychosocial issues are predominately measured in terms of caregiver stress ${ }^{80}$ or satisfaction. ${ }^{32}$ Including multiple dimensions of patients' psychosocial resources as independent variables and broader psychological therapeutic outcome assessments, however, could enhance both theories and the practice of community neurorehabilitation.

Along with advancing the evidence base for community neurorehabilitation, consistent examination of structure, process, and outcome in rehabilitation would lead to improvements in the overall quality of rehabilitation care. Because neurological disease outcomes are highly variable, a lack of effect from an intervention is not unexpected and does not necessarily lead to questions of the quality of the therapeutic approach. ${ }^{46}$ As Cope notes, "deviant or substandard treatment is more easily camouflaged." Structure and process descriptions, however, could identify whether individual and overall program protocols are consistent with current theories and practice norms.

A further benefit of analyses of the structure and process of care is the production of more cohesive neurorehabilitation "teams." One of the few discussions of the obstacles to multidisciplinary community program implementation notes that many staff experienced confusion regarding their roles, despite having contracts for their services. Sheriff and Chenoweth ${ }^{80}$ suggest establishing the process of care is not necessarily straightforward, and may require clarification of expectations prior to data collection. Furthermore, these authors attribute the program's ultimate success to staff commitment and communication. "It was also important for members to trust and respect the concerns, difficulties, and ideas of other members, encourage and engage in democratic de- cision making, and take unified action in decision making." 80 Thus, self-analyses of structure and process could help community neurorehabilitation teams develop truly "interdisciplinary" collaboration. ${ }^{81}$

\section{CONCLUSIONS}

Community neurorehabilitation, especially early supported discharge and goal-oriented physical and occupational therapies, can lead to improvements in activities of daily living after the onset of a range of neurological conditions. These findings suggest a need for thorough examination of current assumptions regarding the limits on functional recovery in community settings. Further research is required to determine more precise therapeutic thresholds for neurological disorders among individuals living in the community and the impact of community-based "maintenance" therapies. Attention to structure, process, and quality of life outcome measures in community neurorehabilitation clinical trials, as well as patient level variables, would help further refine understanding of the elements of multidisciplinary rehabilitation associated with the greatest recovery.

Acknowledgments: This research was supported in part by the Department of Veterans Affairs Rehabilitation Research and Development Program. The author thanks Michael Weinrich and the anonymous reviewers for their comments.

\section{REFERENCES}

1. Thom T, Haase N, Rosamond W, et al. Heart Disease and Stroke Statistics - 2006 Update. Circulation 2006;113:e85-e151.

2. Langlois J, Rutland-Brown W, Thomas K. Traumatic brain injury in the United States: emergency department visits, hospitalizations, and deaths. Atlanta, GA: Centers for Disease Control and Prevention, National Center for Injury Prevention and Control; 2004.

3. Office of Communications and Public Liaison, NINDS. Parkinson's disease: hope through research. Available at: http://www. ninds.nih.gov/disorders/parkinsons_disease/detail_parkinsons_ disease.htm. Accessed Date: May 18, 2006.

4. National Parkinson Foundation. About Parkinson disease. Available at: http://www.parkinson.org/site/apps/s/content.asp?c= 9dJFJLPwB\&b=108269\&ct $=89590$. Accessed Date: May 18, 2006.

5. Office of Communications and Public Liaison, NINDS. Multiple sclerosis: hope through research. Available at: http://www.ninds. nih.gov/disorders/multiple_sclerosis/detail_multiple_sclerosis.htm. Accessed Date: May 18, 2006.

6. Hankey GJ, Langhorne P. Services for reducing the duration of hospital care for acute stroke patients. Stroke 2006;37:276-277.

7. Langhorne P, Taylor G, Murray G, et al. Early supported discharge services for stroke patients: a meta-analysis of individual patients' data. Lancet 2005;365:501-506.

8. Barnes MP, Radermacher H. Neurological rehabilitation in the community. J Rehabil Med 2001;33:244-248.

9. Brown TR, Kraft GH. Exercise and rehabilitation for individuals with multiple sclerosis. Phys Med Rehabil Clin N Am 2005;16: 513-555.

10. Watson C, Rutterford NA, Shortland D, Williamson N, Alderman N. Reduction of chronic aggressive behaviour 10 years after brain injury. Brain Inj 2001;11:1003-1015.

11. Outpatient Service Trialists. Therapy-based rehabilitation for stroke patients living at home. Cochrane Database Syst Rev 2003; (1):CD002925 (Online). 
12. Rietberg MB, Brooks D, Uitdehaag BMJ, Kwakkel G. Exercise therapy for multiple sclerosis. Cochrane Database Syst Rev 2005; 25:CD003980 (Online).

13. Wade D. Community rehabilitation, or rehabilitation in the community? Disabil Rehabil 2003;25:875.

14. Wade DT, de Jong BA. Recent advances in rehabilitation. BMJ 2000;320:1385-1388.

15. World Health Organization. Towards a common language for functioning, disability and health: ICF. Geneva: World Health Organization; 2002.

16. Kraft GH, Freal JE, Coryell JK. Disability, disease duration, and rehabilitation service needs in multiple sclerosis: patient perspectives. Arch Phys Med and Rehab 1986;67:164-168.

17. Anderson C, Ni Mhurchu C, Brown PM, Carter K. Stroke rehabilitation services to accelerate hospital discharge and provide home-based care: an overview and cost analysis. Pharmacoeconomics 2000;20:537-552.

18. Cott C. Client-centred rehabilitation: client perspectives. Disabil Rehabil 2004;26:1411-1422.

19. Thompson AJ. Neurorehabilitation in multiple sclerosis: foundations, facts and fiction. Curr Opin Neurol 2005;18:267-271.

20. Freeman JA, Hobart JC, Thompson AJ. Outcomes-based research in neurorehabilitation: the need for multidisciplinary team involvement. Disabil Rehabil 1996;18:106-110.

21. Ashburn A, Jones D, Plant R, et al. Physiotherapy for people with Parkinson's disease in the UK: an exploration of practice. Int $\mathrm{J}$ of Ther Rehabil 2004;11:160-166.

22. Geddes JML, Chamberlain MA. Home-based rehabilitation for people with stroke: a comparative study of six community services providing coordinated, multidisciplinary treatment. Clin Rehabil 2001;15:589-599.

23. Makepeace RW, Barnes MP, Semlyen JK, Stevenson J. The establishment of a community multiple sclerosis team. Int J Rehabil Res 2001;24:137-141.

24. Enderby P, Wade DT. Community rehabilitation in the United Kingdom. Clin Rehabil 2001;15:577-581.

25. McMillan TM, Ledder H. A survey of services provided by community neurorehabilitation teams in South East England. Clin Rehabil 2001;15:582-588.

26. Steultjens E, Dekker J, Bouter L, van de Nes J, Cup E, van den Ende C. Occupational therapy for stroke patients: a systematic review. Stroke 2003;34:676-687.

27. Wade DT. Community rehabilitation. Clin Rehabil 2001;15:575.

28. Turner-Stokes L, Disler PB, Nair A, Wade DT. Multi-disciplinary rehabilitation for acquired brain injury in adults of working age. Cochrane Database Syst Rev 2005;20:CD004170 (Online).

29. Gage H, Storey L. Rehabilitation for Parkinson's disease: a systematic review of available evidence. Clin Rehabil 2004;18:463482.

30. Legg L, Langhorne P, Outpatient Service Trialists. Outpatient Service Trialists. Rehabilitation therapy services for stroke patients living at home: systematic review of randomized trials. Lancet 2004;363:352-356.

31. Legg L, Langhorne P. Therapy-based rehabilitation for stroke patients living at home. Stroke 2004;35:1022.

32. Early Supported Discharge Trialists. Services for reducing duration of hospital care for acute stroke patients. Cochrane Database of Syst Rev 2005;18:CD000443 (Online). 2005.

33. Walker MF, Leonardi-Bee J, Bath P, et al. Individual patient data meta-analysis of randomized controlled trials of community occupational therapy for stroke patients. Stroke 2004;35:2226-2232.

34. Van Peppen RPS, Kwakkel G, Wood-Dauphinee S, Hendriks HJM, Van der Wees PJ, Dekker J. The impact of physical therapy on functional outcomes after stroke: what's the evidence? Clin Rehabil 2004;18:833-862.

35. Pang MYC, Eng JJ, Dawson AS, Gylfadottir S. The use of aerobic exercise training in improving aerobic capacity in individuals with stroke: a meta-analysis. Clin Rehabil 2006;20:97-111.

36. Langhorne P, Dennis MS, Kalra L, Shepperd S, Wade DT, Wolfe CD. Services for helping acute stroke patients avoid hospital admission. Cochrane Database Syst Rev 2000;(2):CD000444. Review.
37. Kraft GH. Rehabilitation principles for patients with multiple sclerosis. J Spinal Cord Med 1998;21:117-120.

38. Kesselring Jr. Rehabilitation in multiple sclerosis. ACNR 2002;2: $6-8$.

39. Kesselring Jr, Beer S. Symptomatic therapy and neurorehabilitation in multiple sclerosis. Lancet Neurol 2005;4:643-652.

40. Patti F, Ciancio MR, Cacopardo M, et al. Effects of a short outpatient rehabilitation treatment on disability of multiple sclerosis patients: a randomized controlled trial. J Neurol 2003;250:861866.

41. Di Fabio RP, Soderberg J, Choi T, Hansen CR, Schapiro RT. Extended outpatient rehabilitation: its influence on symptom frequency, fatigue, and functional status for persons with progressive multiple sclerosis. Arch Phys Med Rehabil 1998;79:141-146.

42. Steultjens EMJ, Dekker J, Bouter LM, et al. Occupational therapy for multiple sclerosis. Cochrane Database Syst Rev 2003;(3): CD003608.

43. Kesselring J. Rehabilitation in MS is effective. Intl Multiple Sclerosis J 2001;8:68-71.

44. Petajan JH, White AT. Recommendations for physical activity in patients with multiple sclerosis. Sports Med 1999;27:179-191.

45. Mazaux JM, Richer E. Rehabilitation after traumatic brain injury in adults. Disabil Rehabil 1998;20:435.

46. Cope DN. The effectiveness of traumatic brain injury rehabilitation: a review. Brain Inj 1995;9:649-670.

47. Chesnut RM, Carney N, Maynard H, Mann NC, Patterson P, Helfand M. Summary report: evidence for the effectiveness of rehabilitation for persons with traumatic brain injury. J Head Trauma Rehabil 1999;14:176-188.

48. Brooks N. The effectiveness of post-acute rehabilitation. Brain Inj 1994;5:103-109

49. Thompson AJ, Playford ED. Rehabilitation for patients with Parkinson's disease. Lancet 2001;357:410.

50. Deane KHO, Ellis-Hill C, Jones D, et al. Systematic review of paramedical therapies for Parkinson's disease. Mov Disord 2002; 17:984-991

51. Lim I, van Wegen E, de Goede C, et al. Effects of external rhythmical cueing on gait in patients with Parkinson's disease: a systematic review. Clin Rehabil 2005;19:695-713.

52. Murphy S, Tickle-Degnen L. The effectiveness of occupational therapy-related treatments for persons with Parkinson's disease: a meta-analytic review. Am J Occup Ther 2001;55:385-392.

53. Trend P, Kaye J, Gage H, Owen C, Wade D. Short-term effectiveness of intensive multidisciplinary rehabilitation for people with Parkinson's disease and their carers. Clin Rehabil 2002;16:717725.

54. Wade DT, Gage H, Owen C, Trend P. Multidisciplinary rehabilitation for people with Parkinson's disease: a randomized controlled study. J Neurol Neurosurg Psychiatry 2003;74:158-162.

55. Anderson C, Rubenach S, Mhurchu CN, Clark M, Spencer C, Winsor A. Home or hospital for stroke rehabilitation? results of a randomized controlled trial: I: health outcomes at 6 months. Stroke 2000;31:1024-1031.

56. Anderson C, Mhurchu CN, Rubenach S, Clark M, Spencer C, Winsor A. Home or hospital for stroke rehabilitation? results of a randomized controlled trial: II: cost minimization analysis at 6 months. Stroke 2000;31:1032-1037.

57. Bode RK, Heinemann A, Semik P, Mallinson T. Relative importance of rehabilitation therapy characteristics on functional outcomes for persons with stroke. Stroke 2004;35:2537-2542.

58. Ottenbacher K. The Post-Stroke Rehabilitation Outcomes Project. Stroke 2005;86:S121-S123.

59. Horn S, DeJong G, Smout R, Gassaway J, James R, Conroy B Stroke rehabilitation patients, practice, and outcomes: is earlier and more aggressive therapy better? Arch Phys Med Rehab 2005;86: S101-S114.

60. Kwakkel G, Van Peppen RPS, Wagenaar RC, et al. Effects of augmented exercise therapy time after stroke: a meta-analysis. Stroke 2004;35:2529-2539.

61. Ryan T, Enderby P, Rigby A. A randomized controlled trial to evaluate intensity of community-based rehabilitation provision following stroke or hip fracture in old age. Clin Rehabil 2005;20: 123-131. 
62. DeJong G, Horn S, Conroy B, Nichols D, Healton E. Opening the black box of poststroke rehabilitation: stroke rehabilitation patients, processes, and outcomes. Arch Phys Med Rehabil 2005;86: S1-S7.

63. Donabedian A. Evaluating the quality of medical care. Milbank Q 2005;83:691-729

64. Larson J, Muller A. Managing the quality of health care. J Health Hum Serv Adm 2002;25:261-280.

65. Hoenig H, Sloane R, Horner RD, Zolkewitz M, Duncan PW, Hamilton BB. A taxonomy for classification of stroke rehabilitation services. Arch Phys Med Rehabil 2000;81:853-862.

66. Klonoff PS, Lamb DG, Henderson SW, Shepherd J. Outcome assessment after milieu-oriented rehabilitation: new considerations. Arch Phys Med Rehabil 1998;79:684-690.

67. McCorkel BA, Glueckauf RL, Ecklund-Johnson EP, Tomusk AB, Trexler LE, Diller L. Development and exploratory analysis of the neurorehabilitation program styles survey. J Head Trauma Rehabil 2003; $18: 425$

68. Hoenig H, Duncan PW, Horner RD, et al. Structure, process, and outcomes in stroke rehabilitation. Med Care 2002;40:1036-1047.

69. Hart E. System induced setbacks in stroke recovery. Sociol Health Illn 2001; 23:101-123.

70. Logan P, Gladman JRF, Drummond A, Radford KA. A study of interventions and related outcomes in a randomized controlled trial of occupational therapy and leisure therapy for community stroke patients. Clin Rehabil 2003;17:249-255.

71. Kelly-Hayes M. Stroke outcome measures. J Cardiovasc Nurs 2004;19:301-307.
72. Kwon S, Hartzema A, Duncan PW, Min-Lai S. Disability measures in stroke: relationship among the Barthel Index, the Functional Independence Measure, and the Modified Rankin Scale. Stroke 2004;35:918-923.

73. Boylstein C, Rittman M. The importance of narratives in stroke rehabilitation. Generations 2003;27:49-54.

74. Cicerone KD, Mott T, Azulay J, Friel JC. Community integration and satisfaction with functioning after intensive cognitive rehabilitation for traumatic brain injury. Arch Phys Med Rehabil 2004; 85:943-950.

75. Boden-Albala B, Litwak E, Elkind M, Rundek T, Sacco R. Social isolation and outcomes post stroke. Neurology 2005;64: $1888-1892$.

76. Shaughnessy M, Resnick B, Macko R. Testing a model of poststroke exercise behavior. Rehabil Nurs 2006;31:15-21.

77. Resnick B. Efficacy beliefs in geriatric rehabilitation. J Gerontol Nurs 1998; 24:34-44.

78. Watson LD, Quinn DA. Stages of stroke: a model for stroke rehabilitation. Br J Nurs 1998;7:631-640.

79. Barker RN, Brauer SG. Upper limb recovery after stroke: the stroke survivors' perspective. Disabil Rehabil 2005;27:1213-1223.

80. Sheriff JN, Chenoweth L. Challenges in conducting research to improve the health of people with Parkinson's disease and the well-being of their family carers. Int J Rehabil Res 2003;26:201205.

81. Peters MD, McLean A Jr. The evolution of the clinician-scientist model of neurological rehabilitation. Brain Inj 1995;9:543-552. 